Research Journal of Applied Sciences, Engineering and Technology 5(5): 1762-1768, 2013

DOI:10.19026/rjaset.5.4935

ISSN: 2040-7459; e-ISSN: 2040-7467

(C) 2013 Maxwell Scientific Publication Corp.

Submitted: July 31, $2012 \quad$ Accepted: September 03, $2012 \quad$ Published: February 11, 2013

Research Article

\title{
Wind Power Prediction Investigation
}

\author{
${ }^{1}$ Yuanlong Liu, ${ }^{2}$ Yuanbiao Zhang and ${ }^{2}$ Ziyue Chen \\ ${ }^{1}$ Department of Electrical and Information Engineering, \\ ${ }^{2}$ Department of Packaging Engineering, Mathematical Modeling Innovative \\ Practice Base, Jinan University, Zhuhai 519070, China
}

\begin{abstract}
Daily and real-time forecast data of wind power is predicted in this study using three methods, which are Kalman filter model, GARCH model and time-series-based BP neural network model. Then, owing to evaluation to the calculation of accuracy and qualification rate, the best method, the time-series-based BP neural network model, was selected for its highest accuracy. Moreover, the prediction error influence due to convergence of wind turbine is on consideration according to the evaluation. Finally, suggestions of improving the prediction accuracy were put forward based on the discussion of accuracy-obstacle factors.
\end{abstract}

Keywords: BP neutral network, Kalman filter, time sequence prediction, wind-power prediction

\section{INTRODUCTION}

Recently, the main source of wind power generation is the close-to-ground wind, whose volatility, intermittency and low energy density leads to the instability of wind power. The large instability would bring side effects on power balance and frequency regulation of network when a large scale wind farm connects to the public network. Electric power dispatching department could make a schedule to ensure power balance and operation safety, if the wind power could be predicted. As a result, the precise prediction for power in wind farms is a pivotal problem remains settled.

According to the mathematical model, wind power prediction method can be divided into continuous prediction method, Kalman filter model, the ARMA model and intelligence method, etc. Continuous prediction method (Brown et al., 1984) is the most simple prediction model, it assumes that wind speed is equal to the sliding average of the values of the last few wind prediction speed (Lexiadis et al., 1998), but its prediction results are not stable and precise. There are improved method, like Kalman filter (Bossanyi, 1985), ARMA model (Boone, 2005) and time sequence method combined with Kalman filter. There are also some intelligence methods, such as artificial neural network method (Kariniotakis et al., 1996), etc.

In the centralized exploitation mode, the main mode existed of wind power generation, the power of each wind turbine gathers in the wind farm before connecting to the network. The gather of several wind turbines might change the wave characteristics, influencing the prediction error further. The relationship of prediction error between the power of one wind turbine and the total power of several wind turbines remain description, which lead to much more researches needed for the influence that the gathering of wind turbines has on wind power prediction.

\section{WIND POWER PREDICTION}

Due to the different demand in operation mode by electric power dispatching department, prediction of wind power could be divided into two types, daily prediction and real-time prediction. In daily predictions, the 96 time points for wind power of the next $24 \mathrm{~h}$ could be forecasted while 16 time points for the following $4 \mathrm{~h}$. The interval time of two adjacent time points in both predictions is $15 \mathrm{~min}$.

There is a wind farm containing 58 wind turbines, whose rated power output is $850 \mathrm{~kW}$. In this study, wind power of 4 wind turbines $\left(\mathrm{P}_{\mathrm{A}}, \mathrm{P}_{\mathrm{B}}, \mathrm{P}_{\mathrm{C}}, \mathrm{P}_{\mathrm{D}}\right)$ and the total power $\left(\mathrm{P}_{58}\right)$ were predicted by both daily prediction and real-time prediction according to the data from May $10^{\text {th }}$ to June $6^{\text {th }}$ in 2006.

Real-time prediction of wind power based on Kalman filter: For prediction with Kalman filter, prediction recursion equation ought to be deducted using orthogonality theorem by mathematical induction. The following Eq. (1) is the final prediction recursion equation (Bossanyi, 1985):

\footnotetext{
Corresponding Author: Yuanlong Liu, Department of Electrical and Information Engineering, Jinan University, Zhuhai 519070, China
}

This work is licensed under a Creative Commons Attribution 4.0 International License (URL: http://creativecommons.org/licenses/by/4.0/). 


$$
\left\{\begin{array}{c}
\hat{X}(k+1 \mid k+1)=\Phi(k+1, k) \hat{X}(k \mid k)+K(k+1) \\
{[Z(k+1)-H(k+1) \Phi(k+1, k) \hat{X}(k \mid k)]} \\
K(k+1)=P(k+1, k) H^{T}(k+1)[H(k+1) \\
\left.P(k+1 \mid k) H^{T}(k+1)+R(k+1)\right]^{-1} \\
P(k+1 \mid k)=\Phi(k+1, k) P(k \mid k) \Phi^{T}(k+1, k) \\
+\Gamma(k+1, k) Q(k) \Gamma^{T}(k+1, k) \\
P(k+1 \mid k+1)=[I-k(k+1) H(k+1)] \\
P(k+1 \mid k)
\end{array}\right.
$$

In the equation, $\hat{X}(k+1 \mid k+1)$ means the state estimation. $K(k+1)$ means the Kalman gain matrix. $P(k+1 \mid k+1)$ means error covariance matrix predicted by Kalman filter. All the variables above are based on the time point, $k+1 . P(k+1, k)$ is the singlestep prediction-error covariance matrix from the time point $k$ to $k+1 . \mathrm{Q}(k)$ is covariance matrix relating to $\mathrm{w}(k) \cdot \mathrm{R}(k)$ means covariance matrix relating to $\mathrm{v}(k)$. I is the unit matrix. The equations in (1) are optimal filter estimation equation, optimal gain matrix equation, single-step prediction-error covariance matrix equation and error covariance matrix equation predicted by Kalman filter.

Accurate state equation and measuring equation should be deducted as well. To reach the aim, instable differential ARMA model is used in the study, as is shown in (2):

$$
X(k+1)=a_{1} X(k)+a_{2} X(k-1)+a_{3} X(k-2)+a_{4} X(k-3)
$$

In the equation, $a_{1}, a_{2}, a_{3}, a_{4}$ are the coefficient of correspondence.

Four delays are included in (2), which have to be rewrite as a matrix in order to use it in the Kalman filter. Assuming $\mathrm{X}_{1}(k)=\mathrm{X}(k), \quad \mathrm{X}_{2}(k)=\mathrm{X}(k-1)$, $\mathrm{X}_{3}(k)=\mathrm{X}(k-2), \mathrm{X}_{4}(k)=\mathrm{X}(k-3)$, equation could be get as following (3):

$$
\left[\begin{array}{l}
X_{1}(k+1) \\
X_{2}(k+1) \\
X_{3}(k+1) \\
X_{4}(k+1)
\end{array}\right]=\left[\begin{array}{cccc}
a_{1} & a_{2} & a_{3} & a_{4} \\
1 & 0 & 0 & 0 \\
0 & 1 & 0 & 0 \\
0 & 0 & 1 & 0
\end{array}\right]\left[\begin{array}{l}
X_{1}(k) \\
X_{2}(k) \\
X_{3}(k) \\
X_{4}(k)
\end{array}\right]+\left[\begin{array}{l}
1 \\
0 \\
0 \\
0
\end{array}\right] w(k+1)
$$

Obviously, the expression, $\mathrm{Z}(k+1)=\mathrm{X}(k+1)+$ $\mathrm{v}(k+1)$, could be put forward. In the expression, $\mathrm{v}(k+1)$ is the addition noise which is assumed as white noise for the convenience of modeling? The equation for measuring is expressed as the following (4):

$$
Z(k+1)=[1,0,0,0]\left[\begin{array}{l}
X_{1}(k+1) \\
X_{2}(k+1) \\
X_{3}(k+1) \\
X_{4}(k+1)
\end{array}\right]+v(k+1)
$$
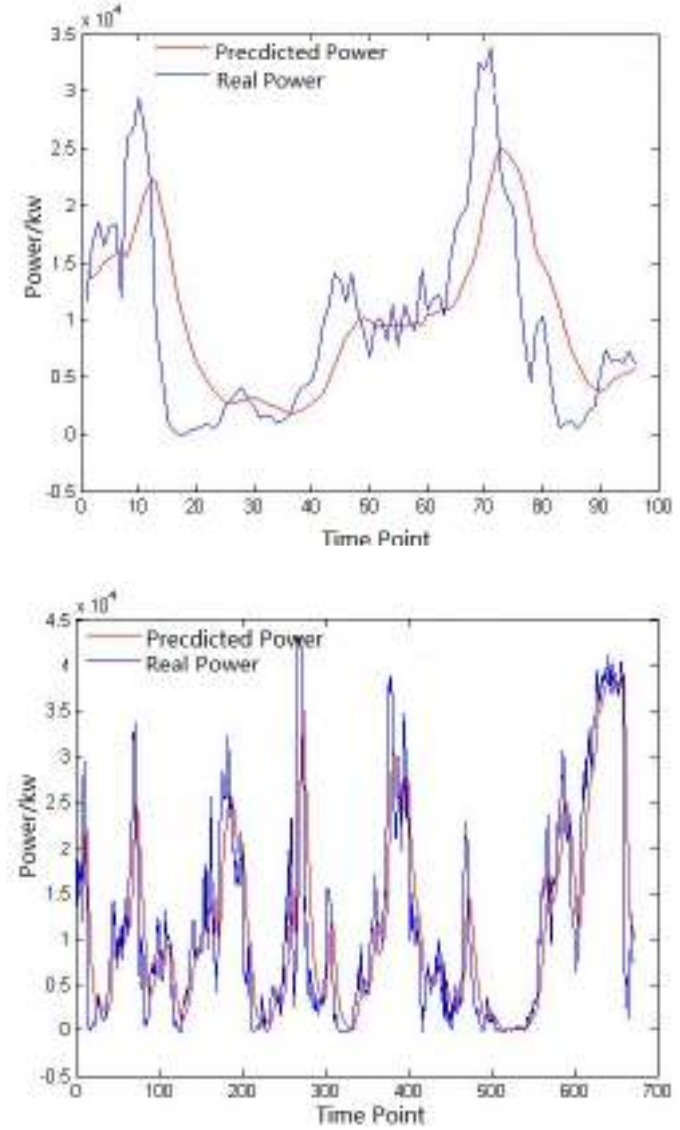

Fig. 1: Predicted and active power graph of two period of time

The occurrence of $\Phi(k+1, k), \Gamma(k+1, k)$ and $H(k+$ 1 could be figured out according to the Eq. (5) and the Eq. (6). After defying the initial state $\mathrm{X}(0 \mid 0)$ and $\mathrm{P}(0 \mid 0)$, recursive equation could be used for iterative prediction with Eq. (1). Considering convergence rate and engineering habits, the initial value is defined as $\mathrm{X}(0 \mid 0)=[0]$ and $\mathrm{P}(0 \mid 0)=101$. MATLAB is used here for the predictive value of wind power with $\mathrm{R}(\mathrm{k})=[1](k=1,2,3 \ldots)$ and $\mathrm{Q}(k)=[1](k=1,2,3 \ldots)$ since it is easy to realize stepwise predict.

Take $\mathrm{P}_{58}$ as an example, the predicted power and the active power of two period of time, from 0:00 to 23:45 in $31^{\text {st }}$ May and from 0:00 to 23:45 in $6^{\text {th }}$ June is shown in Fig. 1.

As is shown in Fig. 1, the real-time prediction of wind power based on Kalman filter enjoys a good effect, which basically reflects the fluctuation tendency of $\mathrm{P}_{58}$ though it cannot predict the fluctuation of large amplitude.

Prediction based on GARCH model:

Construction of the GARCH model (Zhou et al., 2011): GARCH model enjoys the advantages in describing the volatility of time series, especially under 
Res. J. Appl. Sci. Eng. Technol., 5(5): 1762-1768, 2013

the condition that, $\mathrm{q}$, the order of the time series is too big for $\mathrm{ARCH}$ (q) model to describe. GARCH model are often used to overcome low efficiency of iterative parameter, if the sample size is limited. The definition of GARCH model is described as (5):

$$
\left\{\begin{array}{l}
\gamma_{t}=\chi_{t}^{T}+\varepsilon_{t} \\
\varepsilon_{t}=\sigma_{t} v_{t} \quad v_{t} \in \operatorname{NID}(0,1) \\
\sigma_{t}^{2}=a_{0}+a_{1} \varepsilon_{t-1}^{2}+\ldots a_{q} \varepsilon_{t-q}^{2}+\delta_{1} \sigma_{t-1}^{2}+\ldots+\delta_{p} \sigma_{t-p}^{2}
\end{array}\right.
$$

Relationship of the parameters in (5) applies to:

$$
\sum_{i=1}^{q} a_{i}+\sum_{j=1}^{p} \delta_{j}<1, a_{0}>0, a_{i} \geq 0, \delta_{j} \geq 0, i=1,2, \cdots, q, j=1, \cdots, p
$$

Adding the auto-regression module to the expression, $\delta_{t}^{2}$, in $\mathrm{ARCH}$ model, conditional variance in the GARCH model is not only related with the square of the prophase disturbance term but the prophase numerical value of the conditional variance. In fact, low order model are usually used taking place of (5), the expression of GARCH (p, q), as (6), a most frequently used model, GARCH $(1,1)$ :

$$
\begin{aligned}
\sigma_{t}^{2} & =a_{0}+a_{1} \varepsilon_{t-1}^{2}+\delta_{1} \sigma_{t-1}^{2}=a_{1} \varepsilon_{t-1}^{2}+\delta_{1} \sigma_{t-1}^{2}+\delta_{1}\left(a_{0}+a_{1} \varepsilon_{t-2}^{2}+\delta_{1}(\cdots)\right) \\
& =\frac{a_{0}}{1-\delta_{1}}+a_{1}\left(\sigma_{t-1}^{2}+\delta_{1} \sigma_{t-2}^{2}+\delta_{1} \sigma_{t-3}^{2}+\cdots\right)
\end{aligned}
$$

$\sigma_{t}^{2}$, in the GARCH model does not have the characteristic of q-order censoring, which means the unlimited memory to the square of the former error term. The change makes the model much more suitable for the feature of time series in wind-power prediction. The biggest feature of the GARCH model is to describe a complex ARCH model with few parameters, lowering the number of parameters, which bring convenience for parameter estimation and parametric test.

Power prediction: Wind-power data of $31^{\text {st }}$ June are predicted based on the data of wind turbine A from $28^{\text {th }}$ to $30^{\text {th }}$ June since the least sample that GARCH request is 200 as usual.

Stationary analysis: Figure 2 is the unit root test result of the data using EVIEWS 5.1. As is shown in the figure, the t-Statistic of the time series is larger than each ethical value of three significance levels. Thus, the raw time series are non-stationary. Based on the condition, first order difference of the raw data is calculated and the stationary test of the new data meet

\begin{tabular}{lccc}
\hline \hline & t-Statistic & Prob. $^{*}$ \\
\hline \hline Augmented Dickey-Fuller test statistic & -2.470509 & 0.1238 \\
\hline Test critical values: & $1 \%$ level & -3.453234 & \\
& $5 \%$ level & -2.871510 & \\
& $10 \%$ level & -2.572154 & \\
\hline \hline
\end{tabular}

Fig. 2: $\mathrm{t}$-statistic of the data series

\begin{tabular}{ccccc}
\hline \hline \multicolumn{1}{c}{ Variable } & Coefficient & Std. Error & t-Statistic & Prob. \\
\hline \hline C C & 187.9585 & 34.42464 & 5.459999 & 0.0000 \\
AR(1) & 0.829172 & 0.033299 & 24.90119 & 0.0000 \\
\hline \hline R-squared & 0.685107 & Mean dependent var & 180.6096 \\
Adjusted R-squared & 0.684002 & S.D. dependent var & 177.0023 \\
S.E. of regression & 99.49952 & Akaike info criterion & 12.04513 \\
Sum squared resid & 2821544. & Schwarz criterion & 12.07063 \\
Log likelihood & -1726.476 & F-statistic & 620.0693 \\
Durbin-Watson stat & 2.457524 & Prob(F-statistic) & 0.000000 \\
\hline \hline Inverted AR Roots & \multicolumn{2}{c}{} & &
\end{tabular}

Fig. 3: Related parameter estimation and statistic value of AR (1)

\begin{tabular}{llll}
\hline \hline F-statistic & 31.39198 & Prob. Fit 284 & 9.000000 \\
Obs'Risquared & 28.41750 & Prob. ChiSquare[1] & 0.000000 \\
\hline \hline
\end{tabular}

Fig. 4: Residual tested by Lagrangian multiplier method

the request. As a result, the non-stationary of the raw data is weak.

Establishment of the ARCH model: According to the establishing steps of the ARCH model, the basic form of the time series is figured out. Then, residual is tested by Lagrangian multiplier method to figure out whether $\mathrm{ARCH}$ effect exists in the time series. If the ARCH effects do exist, related $\operatorname{AR}(k)$ and $\operatorname{GARCH}(\mathrm{p}, \mathrm{q})$ model would be established based on correlation effect and the general form of GARCH model.

According to the analysis above, raw data could be fitted by $\operatorname{AR}(k)$ and $\operatorname{GARCH}(\mathrm{p}, \mathrm{q})$. Auto-correlation module of time series is described in AR $(k)$. As autocorrelation module of the time series is usually less than 5, 5 models, which are expressed as AR (1), AR (2), AR (3), AR (4) and AR (5), are established. If ARCH effect exists in $\operatorname{AR}(k)$, build $\operatorname{GARCH}(1,1)$ and GARCH $(2,2)$ model for its residual. Taking AR (1) as an example, related parameter estimation and statistic value is calculated and expressed in Fig. 3 by EVIEWS 5.1 .

Testing the residual of AR (1) with Lagrangian multiplier method, the following results are figured out as is shown in Fig. 4.

In Fig. $4 L M=T R^{2}=28.4175>x_{0.05}^{2}=3.841$. Since theLM -related probability is smaller than 0.05 , the value is in the right side of critical value, which is the rejection region of null hypothesis. As a result, $\mathrm{ARCH}$ exists in three error sequence of the model. 


\begin{tabular}{|c|c|c|c|c|}
\hline & Cooficiant & Std Emai & z-Stakistic & Prob: \\
\hline C & 125.2052 & $44056 \pi 7$ & 2.8546013 & 0. 0042 \\
\hline AR[1) & 0.844319 & 0.044574 & 18.94212 & 0.0000 \\
\hline \multicolumn{5}{|c|}{ Variance Equation } \\
\hline c & 1790za9 & 1433.439 & 12.49946 & 0.0000 \\
\hline FESD면 2 & 0.096717 & 0.025696 & 3324231 & 0.0009 \\
\hline GARCHI-1) & .0840434 & 0.050457 & -16.55318 & 0.0000 \\
\hline R-squared & 0.681835 & \multicolumn{2}{|c|}{ Mean dependant va } & 1BD 6096 \\
\hline Adiusted R-aquared & 0.677322 & \multicolumn{2}{|c|}{ g. D. dependert var } & $1 \pi 7.0023$ \\
\hline S.E of regression & $100545 B$ & \multirow{2}{*}{\multicolumn{2}{|c|}{$\begin{array}{l}\text { Akalice info critorian } \\
\text { Schmarz critorion }\end{array}$}} & 1197413 \\
\hline Sum squared resid & $2 a 50 B 55$ & & & 1203789 \\
\hline Log Sikolinood & $-17132 \mathrm{aB}$ & \multicolumn{2}{|c|}{ F-statistic } & 151.0329 \\
\hline Durbin-Watson stat & 2470618 & \multicolumn{2}{|c|}{ Proesf-statiatic| } & 0.000000 \\
\hline
\end{tabular}

Fig. 5: Related parameter estimation and statistic value of AR (1)-ARCH $(1,1)$

Table 1: Same-model testing results

\begin{tabular}{|c|c|c|c|c|c|}
\hline $\begin{array}{l}\text { AR(k)-GARCH } \\
(\mathrm{p}, \mathrm{q})\end{array}$ & $(1,1,1)$ & $(1,2,1)$ & $(2,1,1)$ & $(2,2,1)$ & $(2,1,2)$ \\
\hline $\mathrm{AIC}$ & 11.974 & 11.743 & 11.623 & 11.821 & 11.921 \\
\hline SIC & 12.038 & 11.921 & 11.745 & 11.825 & 12.127 \\
\hline
\end{tabular}

According to the GARCH $(1,1)$ model of residue based on the AR (1) -mean-value equation, the result could be figured out as Fig. 5.

Thus, the mean-value equation could be figured out as (7):

$$
X_{t}=126.2052+0.844319 X_{t-1}+\varepsilon_{t}
$$

The GARCH equation is:

$$
\sigma_{t}^{2}=17902.89+0.09877 \varepsilon_{t-1}^{2}-0.840434 \sigma_{t-1}^{2}
$$

Therefore, different LM are established for testing different AR ( $k$ )-GARCH (p, q) model.

Model selection: According to the judgment and testing results, 5 models suitable for time series of wind power could be established. Then, different models would be selected based on several criterions. As a result, AIC information criterion and SIC information criterion are chosen for reflecting goodness of fit of time series. As is shown in Table 1, AR (2)-GARCH (1, 1) enjoys the best imitative effect.

Model prediction: Predicting the wind power of $31^{\text {st }}$ June produced by Wind Turbine A by AR (2)-GARCH $(1,1)$, the results are constructed as Fig. 6 . What could be seen from Fig. 6 is cluster effect of the wind-power random perturbation? That means large fluctuation range are often followed with the range of large function and it is the same as that of small fluctuation range, which means the feature of ARCH model. Other results could be figured out with the same principle.

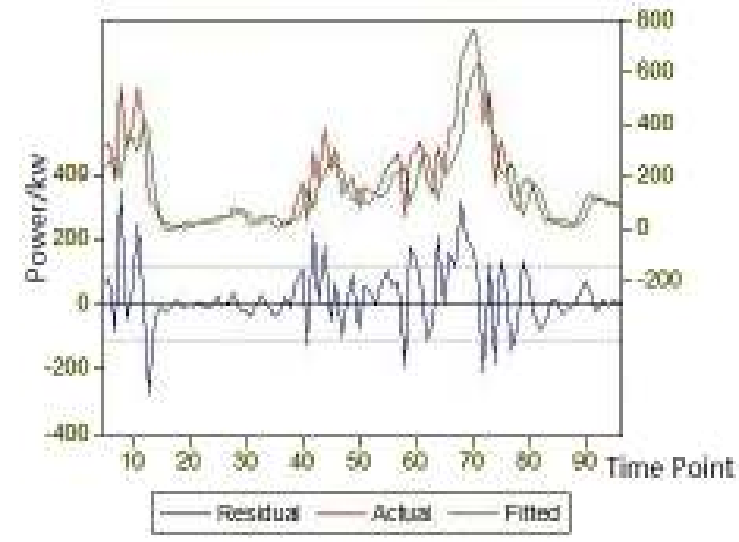

Fig. 6: Wind-power prediction result of $31^{\text {st }}$ June

\section{Real-time prediction by time-series-based BP neural network:}

Establishment of the BP-neural-network prediction model (Zhang and Ghao, 2003):

Deciding network layer: BP neural network is constituted by input layer, output layer and several hidden layer. Each layer is made by several nodes, each of which represents a neuron. Nodes between layers are connected by full-associated connecting mode; no connection is made among nodes within the same layer though. Thus, the number of layer of BP neural network depends on the number of the hidden layers.

Adding the hidden layers cannot meet the request of real-time prediction, since it will increase the calculating time. As a solution to the problem, onehidden-layer BP neural network are chosen to predict the wind power.

Deciding the neuronal number in each layer: There is a close connection between input node, output node and sample, which related with their application field. According to the request of the prediction, wind farm are required to forecast the wind power data every 15 min of the next $4 \mathrm{~h}$. The neural number of input layer is 16, which means 16 wind power data should be conducted as input variable. Meanwhile, the neural number of the output layer is 1 , representing the $17^{\text {th }}$ wind power data. That means the times series, $\mathrm{x}_{1}, \mathrm{x}_{2}$, $\ldots . \mathrm{x}_{\mathrm{n}}$, satisfy with Eq. (8) as following:

$$
x_{i+16}=f\left(x_{i}\right) x_{i}=\left(x_{i}, x_{i+1}, x_{i+2}, \cdots, x_{i+15}\right)
$$

Two points for attention for deciding the neural number of hidden layers is mentioned. The too small neural number will lead to the hardship of recognizing samples, which makes it training obstacle and even lower the network tolerance: 


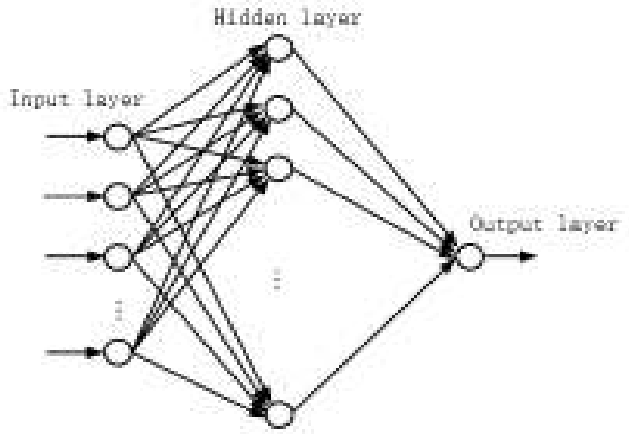

Fig. 7: BP neural network

$$
i=\sqrt{n+m}+k
$$

$\mathrm{i}=$ The neural number of the hidden layer

$\mathrm{n}=$ The neural number of the input layer

$\mathrm{m}=$ The neural of the output layer

Thus, the neural number of the hidden layer is 8 ; the model can be described as Fig. 7.

Training BP neural network by sample vector: Usually, the weight of BP neural network would not change once decided. In this model, wind power is fluctuated since the wind power depends on wind speed, whose feature is volatility, intermittency and low energy density. Thus, weights are adjusted with the following steps:

Step 1: After training the network with the training sample data, $\mathrm{x}_{1}, \mathrm{x}_{2}, \mathrm{x}_{3} \ldots \ldots \mathrm{x}_{16}, \mathrm{x}_{17}$, could be predicted.

Step 2: Take $x_{17}$ as the input sample of the series, which means the training sample of the second group is $\mathrm{x}_{2}, \mathrm{x}_{3} \ldots \ldots$. Using the data to retraining the network, $\mathrm{x}_{18}$ is predicted.

Step 3: After the repeated iteration that training the network with the data, $\mathrm{x}_{\mathrm{t}}, \mathrm{x}_{\mathrm{t}+1} \ldots \ldots \mathrm{x}_{\mathrm{t}+15}$ and predict $x_{t+16}$, the weights of the network tend to be higher precision, which better reflects the change of wind power.

Step 4: Preprocessing data: Number the data from $10^{\text {th }}$ to $30^{\text {th }}$ in May in order of time step. Then, normalizing the number to real number in the interval, $[0,1]$, since the variable range is large. The normalization is expressed as (10):

$$
X_{i}=\frac{x_{i}-x_{\min }}{x_{\max }-x_{\min }}
$$

where,

$\mathrm{X}_{\mathrm{i}}$ : The normalized wind power

$\mathrm{x}_{\mathrm{i}}$ : The original wind power
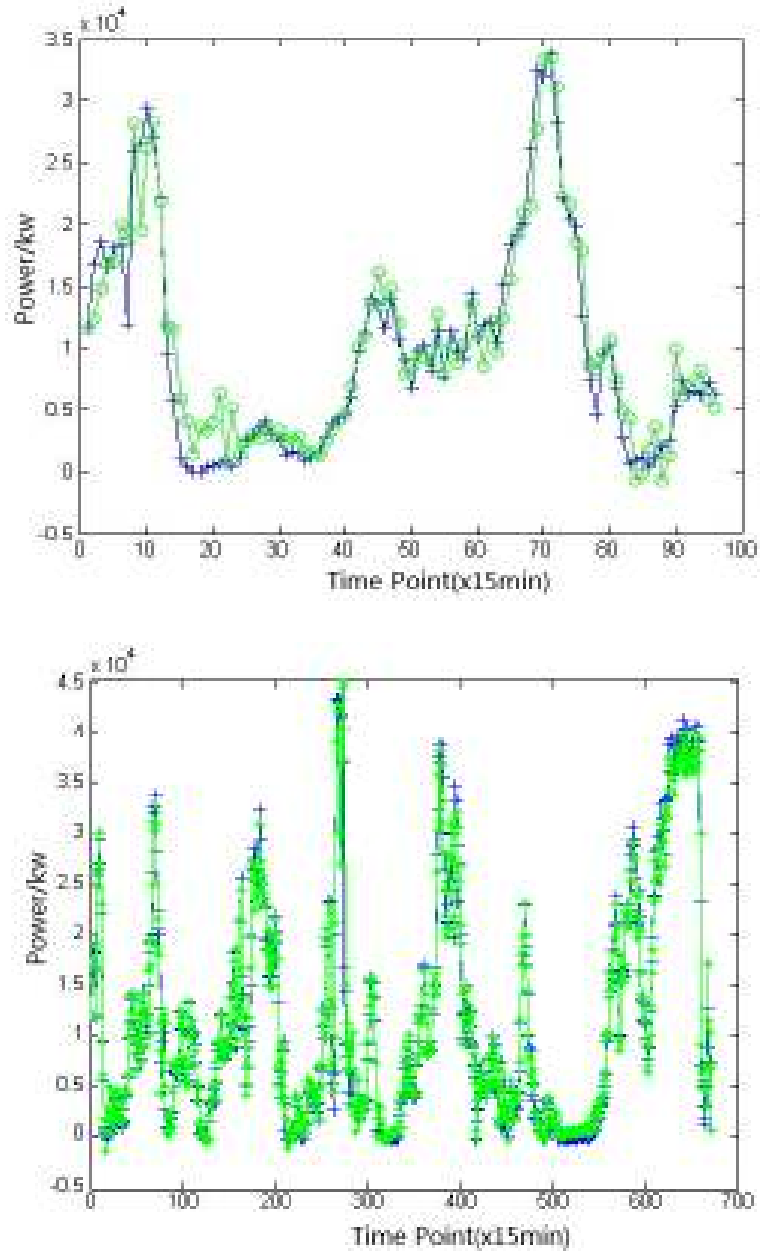

Fig. 8: Predicted and real data of two period of time

$\mathrm{x}_{\max }$ : The maximum of the original wind power

$\mathrm{x}_{\min }$ : The minimum of the original wind power

Step 5: Input the preprocessed number from $10^{\text {th }}$ to $30^{\text {th }}$ May as training sample. The desired error is reached with the iteration number of 392 .

Real-time prediction for wind power with BP neural network: Inputting the historic data to the trained network, 16 wind power data of the following $4 \mathrm{~h}$ could be predicted. Then, the wind power data of $31^{\text {st }}$ May and that from $31^{\text {st }}$ May to $6^{\text {th }}$ June are calculated. Predicted data of wind power from 0:00 to 23:45 and from 0:00 $31^{\text {st }}$ May to 23:45 $6^{\text {th }}$ June could be calculated with MATLAB, which is shown in Fig. 8.

Method evaluation: Accuracy and qualification are established for comparison of the three methods in this study according to relevant standard. 
Res. J. Appl. Sci. Eng. Technol., 5(5): 1762-1768, 2013

Table 2: Accuracy and qualification of the three methods

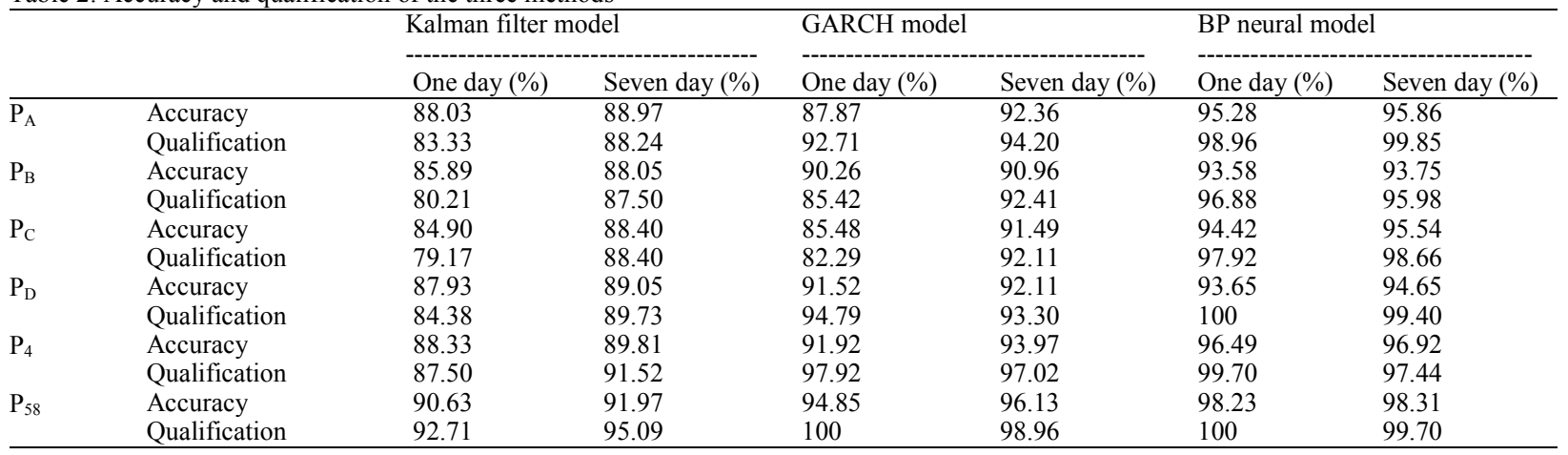

Accuracy: Define the difference between 1 and rootmean-square error as the accuracy $r_{1}$ as (11):

$$
r_{1}=\left(1-e_{\mathrm{RMSE}}\right) \times 100 \%
$$

$\mathrm{e}_{\mathrm{RMSE}}$ is the root-mean-square error.

Qualification: Define qualification as (12):

$$
\left\{\begin{array}{l}
r_{2}=\frac{1}{n} \sum_{i=1}^{n} B_{i} \times 100 \% \\
\frac{\left|e_{i}\right|}{P} \leq 25 \%, \quad B_{i}=1 \\
\frac{\left|e_{i}\right|}{P}>25 \%, \quad B_{i}=0
\end{array}\right.
$$

$\mathrm{e}_{\mathrm{i}}=$ Prediction error

$\mathrm{P}=$ The rated power of the wind farm

Substituting real power data and real power data calculated by the three methods to (11) and (12), accuracy and qualification within one day (from 0:00 to 23:45 $31^{\text {st }}$ May) and seven days (from 0:00 $31^{\text {st }}$ May to $23: 456^{\text {th }}$ June) could be figured out, which is shown in Table 2.

According to the comparison, it is obvious that the time-series-based BP neural network model and GARCH prediction model is better than Kalman filter model. Moreover, the time-series-based BP neural network enjoys more advantages than GARCH prediction model. As a result, the time-series-based BP neural network model is selected for prediction.

\section{PREDICTION ERROR EXPECTATION BY GATHERING}

Based on the analysis above, the law we hope to get is that the gathering of wind turbines obviously decreases the prediction error.
Table 3: Root-mean-square error of different method

\begin{tabular}{llll}
\hline Time step/min & Kalman filter & GARCH & BP neural network \\
\hline 15 & 0.167 & 0.154 & 0.072 \\
30 & 0.174 & 0.165 & 0.082 \\
60 & 0.187 & 0.177 & 0.090 \\
\hline
\end{tabular}

Combining with the facts, wind power are affected by wind speed, wind direction, temperature, sir pressure and so on. The wind power would suffer fluctuation owing to the natural factors above. The fluctuation range enlarges as the range of these factors increase, which are easy to be ignored in the prediction, leading directly to the predicting error. Meanwhile, as wind turbines are built in the same area, the influence of natural factors on wind turbines are similar. After gathering, accumulation of wind-power fluctuation is scaled up with more wind turbines. As a result, the fluctuations that ignored in the single-turbine prediction would be predicted efficiently, lower its predicting error.

At present, wind power is centralized developed. Wind power of each turbine gathers before connecting to the power network by wind farms. It is meaningful for ensuring the power balance and running safety to predict wind power of wind farms accurately. According to the results analyzed, suggestions are made that more wind turbines connect to the power network.

\section{MAIN FACTORS PREVENTING THE RAISE OF THE REAL-TIME PREDICTION}

Number of historic data: Sensitivity analysis is made for analyzing the error by changing the number of historic data. As different numbers of data make contributions to different predicting errors, lacking historic data prevents the improvement of the windpower-prediction accuracy.

Method selection: Different method contributes to different error. If features of the high-accuracy method are find out, accuracy could be improved. 
Selection of time step: Predicting with different time steps, make the sensitivity analysis and error analysis. Then the root-mean-square error of predicted data in $31^{\text {st }}$ June is in Table 3. Root-mean-square error of every model grows when the time step enlarges. Thus, the time step has effect on accuracy of prediction.

\section{CONCLUSION}

Change law of the wind power is well captured by the neural network model, time-series-based GARCH model and Kalman filter model, which all enjoy quantities of advantages in prediction. Among them, Kalman filter keep renewing the weight of state estimation during recursion, ensuring the results close to the real condition. GARCH model not only well considered the sequential characteristic of Root-meansquare error but explain the volatility of time series. The benefit that the neural network has is its characteristics of nonlinear complex signal processing, which greatly approximating continuous curve.

Influenced by similar natural factors, wind powers of turbines in the same area fluctuate much more violently after gathering together, which makes it easier for prediction, leading a higher accuracy.

Due to the existence of systematic errors, accuracy of wind power cannot rise without limitation. However, a more accurate prediction of wind power is of great possibility. Thus, the suggestions are given in the following part based on the factors above.

Firstly, number of the data should be increased and the time step should be narrowed. Secondly, the prediction would be much better developed if meteorological information and physical model are added. In addition, it is tend to build multi-predicting model for forecasting wind power more accurately.

\section{REFERENCES}

Boone, A., 2005. Simulation of Short-Term Wind Speed Forecast Errors Using a Multi-Variate ARMA $(1,1)$ Time-Series Model. Royal Institute of Technology, Sweden, pp: 70-88.

Bossanyi, E.A., 1985. Short-term wind prediction using Kalman filters. Wind Eng., 9: 1-8.

Brown, B.G., R.W. Katz and A.H. Mtlrpby, 1984. Time series models to simulate and forecast wind speed and wind power. J. Clim. Appl. Meteorol., 23(8): 1184-1195.

Kariniotakis, G.N., G.S. Stavrakakis and E.F. Nogaret, 1996. Wind power forecasting using advanced neural networks models. IEEE T. Energy Conver., 11(4): 762-767.

Lexiadis, M.A., P.S. DokoPoulos, S.H. Samanoglou, I.M. Manousaridis, 1998. Short-term forecasting of wind speed and related electrical power. Sol. Energy, 63(1): 61-68.

Zhang, Y. and Z.W. Gao, 2003. The Load Forecasting of Electric Energy Based on Time Sequence and Neural Network. J. Harbin Univ. Sci. Tech., pp: 30-36.

Zhou, H., J.X. Fang and M. Huang, 2011. Application research of wind power forecasting model GARCH. Power Syst. Protect. Control, 39(5): 108-119. 